\title{
Directives de la Société canadienne de thoracologie : Diagnostic et traitement des troubles respiratoires du sommeil de l'adulte
}

\author{
John Fleetham MD, Najib Ayas MD, Doug Bradley MD, Kathy Ferguson MD, Michael Fitzpatrick MD, \\ Charlie George MD, Patrick Hanly MD, Fran Hill TR, John Kimoff MD, Meir Kryger MD, Debra Morrison MD, \\ Frederic Series MD, Willis Tsai MD; Comité des troubles respiratoires du sommeil de la SCT
}

L es directives de la Société canadienne de thoracologie (SCT) concernant le diagnostic et le traitement des troubles respiratoires du sommeil de l'adulte ont été élaborées au cours de l'année écoulée. Une réunion d'un jour s'est tenue à Montréal (Québec) le 28 octobre 2005, juste avant le congrès annuel de la SCT. Cette rencontre a été animée par le docteur R Davies (Oxford, Royaume-Uni), avec des présentations par D Morrison (Halifax, Nouvelle-Écosse), J Kimoff (Montréal), J Fleetham (Vancouver, Colombie-Britannique), C George (London, Ontario), M Kryger (Winnipeg, Manitoba), P Hanly (Calgary, Alberta), F Hill (Saskatoon, Saskatchewan), D Bradley (Toronto, Ontario), N Ayas (Vancouver), M Fitzpatrick (Kingston, Ontario), F Sériès (Québec, Québec), K Ferguson (London) et W Tsai (Calgary). Vingt-huit médecins canadiens s'intéressant aux troubles respiratoires du sommeil y ont pris part. Une ébauche de résumé a été élaborée, puis révisée et finalisée par le Comité des troubles respiratoires du sommeil de la SCT lors d'une journée de réunion à Toronto, le 17 février 2006. Les membres du Comité ont ensuite classé les niveaux de preuve comme suit : niveau A - méta-analyse de qualité ou essai clinique randomisé (ECR) unique comportant un faible risque de biais; niveau $\mathrm{B}$ - analyse méthodique de qualité d'études de cohortes, étude de cohortes unique comportant un faible risque de biais ou preuves extrapolées à partir d'ECR de qualité ou d'ECR comportant un risque de biais; niveau $\mathrm{C}$ études cas-témoins ou études de cohortes comportant un risque de biais; ou niveau $\mathrm{D}$ - séries de cas, exposés de cas ou rapports d'experts. Les membres du Comité ont également indiqué leur opinion concernant chaque déclaration (tout à fait d'accord, d'accord, neutre, pas d'accord ou pas du tout d'accord). Seules les déclarations avec lesquelles au moins 90 $\%$ des membres du comité étaient tout à fait d'accord ou d'accord ont été incluses.

\section{SYNDROMES CLINIQUES ET GRAVITÉ DÉFINITIONS}

Les troubles respiratoires du sommeil (TRS) incluent trois syndromes cliniques distincts, à savoir, le syndrome d'apnéehypopnée obstructive du sommeil (SAHOS), le syndrome d'apnée-hypopnée du sommeil centrale (SAHSC) qui inclut la respiration de Cheyne-Stokes (RCS) et le syndrome d'hypoventilation du sommeil (SHS). Chaque syndrome est assorti de critères diagnostiques distincts.

\begin{abstract}
SAHOS
Critères diagnostiques : L'individu doit répondre au critère $\mathrm{A}$ ou $\mathrm{B}$ et au critère $\mathrm{C}$ (niveau de preuve $\mathrm{D}$ ).

A. Hypersomnie diurne qui ne s'explique pas par d'autres facteurs.

B. Deux ou plusieurs des symptômes suivants qui ne s'expliquent pas par d'autres facteurs :

1. étouffement ou suffocation pendant le sommeil;

2. réveils répétés pendant le sommeil;

3. sommeil non récupérateur;

4. fatigue pendant la journée; et

5. manque de concentration.

C. Un enregistrement réalisé pendant le sommeil révèle cinq apnées/hypopnées obstructives ou davantage par heure de sommeil.
\end{abstract}

Événement d'apnée/hypopnée obstructive : Événement caractérisé par une cessation complète ou une réduction transitoire de la respiration malgré le maintient ou l'augmentation des efforts respiratoires. En pratique clinique courante, il n'est pas jugé nécessaire de distinguer entre les hypopnées et les apnées obstructives, car ces deux types d'événements présentent une physiopathologie similaire. Ces événements doivent répondre au critère $\mathrm{A}$ ou $\mathrm{B}$ et au critère $\mathrm{C}$ (niveau de preuve $\mathrm{D}$ ).

A. Diminution nette (de plus de $50 \%$ de la valeur de base) de l'amplitude du signal de pression nasale ou du signal somme des mouvements thoraco-abdominaux mesurés par pléthysmographie respiratoire par inductance. La valeur de base se définit comme l'amplitude moyenne de la respiration pendant les deux minutes précédant le début de l'événement (chez les individus qui présentent des épisodes de respiration stable pendant le sommeil) ou comme l'amplitude moyenne des trois respirations les plus amples pendant les deux minutes précédant le début de l'événement (chez les individus qui présentent une respiration continuellement instable).

B. Réduction nette de l'amplitude d'une mesure validée de la respiration au cours du sommeil qui n'est pas mentionnée dans le critère précédent mais associée soit à une désaturation en oxygène de $4 \%$ ou davantage, soit à un micro-éveil.

C. L'événement dure au moins 10 secondes.

Comité des troubles respiratoires du sommeil de la SCT

Correspondence: John Fleetham, Respiratory Medicine, 7th Floor, Diamond Health Care Centre, 2775 Laurel Street, Vancouver

(Colombie-Britannique) V5Z 1M9. Téléphone 604-875-5653, télécopie 604-875-5587, courriel John.Fleetham@vch.ca

La version anglaise a été publiée dans le Can Respir J 2006;13(7):387-92 
À l'heure actuelle, il n'existe pas de consensus parmi les spécialistes des TRS en ce qui concerne la définition de l'hypopnée; celle-ci a également été définie comme un événement respiratoire anormal d'une durée minimum de 10 secondes résultant en une réduction d'au moins $30 \%$ du débit respiratoire ou des mouvements thoraco-abdominaux , par rapport à la valeur de base, et s'accompagnant d'une désaturation d'au moins $4 \%$. Les rapports de polysomnographie doivent indiquer clairement la définition retenue de l'hypopnée, voire idéalement comptabiliser séparément les hypopnées selon les deux définitions. Les critères de gravité présentés ci-après se fondent sur la première définition de l'hypopnée, qui n'exige pas une désaturation en oxygène d'au moins $4 \%$ (niveau de preuve $D$ ). Critères de séverité : La gravité du SAHOS inclut deux éléments; la séverité de la somnolence durant la journée et celle des anomalies respiratoires nocturnes. Un degré de gravité doit être spécifié pour chacun de ces éléments. Le taux de gravité du syndrome sera déterminé sur la base de l'élément le plus sévère. A. Somnolence (niveau de preuve D)

1. Légère : Somnolence indésirable ou épisodes de sommeil involontaires pendant des activités qui exigent peu d'attention. Exemples : somnolence devant la télévision, pendant la lecture ou comme passager en voiture.

2. Modérée : Somnolence indésirable ou épisodes de sommeil involontaires pendant des activités qui exigent un peu d'attention. Exemples : somnolence incontrôlable pendant des activités telles qu'un concert, une réunion ou une présentation.

3. Sévère : Somnolence indésirable ou épisodes de sommeil involontaires pendant des activités qui exigent une attention plus active. Exemples : somnolence incontrôlable pendant un repas ou une une conversation, lors de la marche à pied ou au volant d'un véhicule.

B. Indice d'apnée/hypopnée (niveau de preuve B)

1. Léger : Cinq à 15 événements par heure

2. Modéré : 15 à 30 événements par heure

3. Sévère: Plus de 30 événements par heure

\section{SAHSC/RCS}

Ce syndrome inclut l'apnée du sommeil centrale idiopathique (primaire), l'apnée-hypopnée du sommeil centrale due à la RCS, la respiration périodique en haute altitude et l'apnéehypopnée du sommeil centrale secondaire à la prise de médicaments ou à une toxicomanie. Ces deux derniéres conditions se diagnostiquent d'après le contexte approprié et ne seront pas décrits davantage ici.

\section{a) SAHSC}

Critères diagnostiques : L'individu doit répondre aux critères $\mathrm{A}, \mathrm{B}, \mathrm{C}$ et $\mathrm{D}$ (niveau de preuve D).

A. Au moins l'un des symptômes suivants qui ne s'explique pas par d'autres facteurs :

1. hypersomnie ou fatigue pendant la journée; et

2. réveils fréquents la nuit.

B. L'étude du sommeil révèle au moins cinq apnées/hypopnées centrales par heure de sommeil.
C. Normocapnie à l'éveil (tension artérielle partielle de dioxyde de carbone $\left[\mathrm{PaCO}_{2}\right]$ de 35 à $45 \mathrm{mmHg}$ ).

D. Éléments ci-dessus non expliqués par un problème médical, la prise de médicaments ou une toxicomanie.

Événement d'apnée/hypopnée centrale : Événement caractérisé par l'absence ou la réduction du volume courant qui s'accompagne d'une abolition ou d'une diminution des mouvements thoraco-abdominaux. Ces événements doivent répondre aux critères $\mathrm{A}$ et $\mathrm{B}$ (niveau de preuve $\mathrm{D}$ ).

A. Diminution nette (de plus de $50 \%$ de la valeur de base) de l'amplitude la pression nasale ou du signal de pléthysmographie respiratoire par inductance (voir la définition de la valeur de base dans la section précédente relative au SAHOS) avec une réduction proportionnelle des mouvements thoraco-abdominaux.

B. L'événement dure au moins 10 secondes.

Critères de gravité : Il n'existe pas assez de preuves pour proposer des critères de gravité.

\section{b) RCS}

Critères diagnostiques : L'individu doit répondre aux critères A et $B$ (niveau de preuve $D$ ).

A. Présence d'une maladie grave, telle une maladie cardiaque ou neurologique.

B. L'étude du sommeil révèle :

1. au moins cinq apnées/hypopnées centrales par heure de sommeil; et

2. la présence d'une augmentation et diminution cyclique de l'amplitude de la respiration qui peut ou non être assoçiée à des micro-éveils.

Critères de séverité : Il n'existe pas assez de preuves pour proposer des critères de gravité.

\section{SHS}

Critères diagnostiques : L'individu doit répondre aux critères $A$ et $B$ (niveau de preuve $D$ ).

A. L'un ou plusieurs des symptômes suivants :

1. insuffisance cardiaque droite;

2. hypertension artérielle pulmonaire;

3. hypersomnie diurne qui ne s'explique pas par d'autres facteurs;

4. érythrocytose; et

5. hypercapnie à l'éveil $\left(\mathrm{PaCO}_{2}\right.$ supérieure à $45 \mathrm{mmHg}$ ).

B. L'étude du sommeil révèle l'une ou l'autre des anomalies suivantes, voire les deux :

1. une augmentation de la $\mathrm{PaCO}_{2}$ pendant le sommeil de plus de $10 \mathrm{mmHg}$ par rapport aux valeurs mesurées en décubitus dorsal à l'éveil;

2. une hypoxémie prolongée (saturation du sang artériel en oxygène $\left[\mathrm{SaO}_{2}\right]$ de moins de $90 \%$ ) pendant le sommeil, sans apnée ni hypopnée.

Critères de gravité : Le SHS est jugé grave si au moins l'un des critères suivants ( $\mathrm{A}, \mathrm{B}$ ou $\mathrm{C}$ ) est rempli (niveau de preuve $\mathrm{D}$ ). 
A. Hypoxémie à l'éveil (pression partielle d'oxygène $\left[\mathrm{PaO}_{2}\right]$ inférieure à $60 \mathrm{mmHg}$ ou $\mathrm{SaO}_{2}$ inférieure à $90 \%$ ).

B. $\mathrm{SaO}_{2}$ inférieure à $85 \%$ pendant plus de $50 \%$ du temps de sommeil.

C. Insuffisance cardiaque droite, insuffisance bi-ventriculaire ou hypertension artérielle pulmonaire secondaire au SHS.

\section{RÉFÉRENCE EN SOINS SPÉCIALISÉS}

A. Tous les patients chez qui on suspecte un cas de TRS doivent compléter une évaluation de la somnolence diurne, telle l'échelle de somnolence d'Epworth (ESE), afin d'évaluer subjectivement le niveau de somnolence avant le traitement (niveau de preuve D).

B. Les références de patients en centre spécialisé pour évaluation d'un TRS doivent provenir d'un médecin et fournir assez d'informations pour déterminer l'urgence de l'évaluation (niveau de preuve D).

C. Les patients référés à un spécialiste pour une évaluation et/ou une polysomnographie doivent être triés en fonction des catégories et des critères énumérés cidessous (niveau de preuve D).

\section{Priorité 1 (urgent)}

Patients présentant :

- une suspicion de TRS; et

- une somnolence diurne majeure (ESE de 15 ou plus); et

- un travail à haut risque personnel ou pour autrui.

Ou patients présentant :

- une suspicion de TRS; et

- l'un ou plusieurs des symptômes suivants :

- un ou plusieurs facteurs de comorbidité; ou

- une oxymétrie nocturne à domicile qui révèle plus de 30 désaturations en oxygène (4\% ou plus) par heure.

\section{Priorité 2}

Patients présentant :

- une suspicion de TRS; et

- une somnolence diurne majeure (ESE de 15 ou plus); mais

- sans travail à haut risque personnel ou pour autrui.

\section{Priorité 3}

Patients présentant :

- une suspicion de TRS; mais sans :

- somnolence diurne majeure (c.-à-d., ESE de 15 ou plus);

- une ou des comorbidité(s);

- travail à haut risque personnel ou pour autrui.

Comorbidités/grossesse : Cardiopathie ischémique, maladie cérébrovasculaire, insuffisance cardiaque congestive, hypertension artérielle systémique réfractaire, maladie pulmonaire obstructive/restrictive, hypertension artérielle pulmonaire ou insuffisance respiratoire hypercapnique, grossesse.

Travail à haut risque personnel ou pour autrui ou risque élevé de collision avec un véhicule à moteur:
Individus travaillant avec de la machinerie ou effectuant des activités dangereuses; camionneurs, chauffeurs de taxis ou conducteurs d'autobus; mécaniciens de chemin de fer, pilotes de ligne, contrôleurs aériens, mécaniciens d'avions, capitaines et pilotes de navires; et automobilistes qui admettent s'être endormis au volant au cours des deux dernières années. (Tous les patients chez qui on juge qu'ils effectuent une travail à haut risque personnel ou pour autrui doivent être avisés de cesser leurs activités et/ou de ne pas prendre le volant tant que leur évaluation médicale n'a pas été complétée et/ou un traitement approprié mis en place.)

Délais d'attente : L'évaluation par un spécialiste médical et/ou une polysomnographie doivent être organisées et complétées dans les délais suivants après la référence (niveau de preuve $D$ ):

- priorité 1 (urgents) - dans un délai de deux à quatre semaines;

- priorité 2 - dans un délai de deux mois; et

- priorité 3 - dans un délai de six mois.

\section{DIAGNOSTIC}

A. L'étude de niveau I (polysomnographie complète en laboratoire) demeure la norme acceptée pour évaluer la présence de TRS et représente l'enregistrement de choix (niveau de preuve C).

B. Les études de niveau II (polysomnographie ambulatoire complète) et III (polysomnographie ambulatoire dite cardio-respiratoire sans électroencéphalogramme) jouent un rôle utile pour améliorer l'accès au diagnostic des TRS (niveau de preuve C).

C. Les études de niveaux II et III peuvent être utiles pour confirmer le diagnostic de SAHOS chez les patients présentant une probabilité clinique modérée à élevée de cette maladie, mais sont d'une utilité moindre chez les patients présentant des comorbidités. et pour le diagnostic d'autres formes de TRS (niveau de preuve C).

D. Les études utilisant l'oxymétrie seule peuvent jouer un rôle dans l'évaluation initiale des TRS; toutefois, il faut souligner qu'elles ne sont que d'une utilité partielle pour distinguer entre différents types de TRS avant de les utiliser à des fins de diagnostic et de décisions thérapeutiques (niveau de preuve $\mathrm{C}$ ).

E. Le niveau d'expérience et de formation du personnel responsable de l'analyse/interprétation des résultats des études du sommeil est tout aussi important que le type d'étude du sommeil (niveau de preuve D).

F. Toutes les études du sommeil doivent être effectuées dans le cadre d'un programme approprié de contrôle de qualité et être interprétées par un médecin ayant une formation dans la prise en charge des TRS (niveau de preuve D).

G. Les formules de prédiction clinique peuvent être utilisées pour évaluer la probabilité clinique pré-test de TRS et pour établir l'ordre de priorité des patients à évaluer, mais elles ne suffisent pas à établir un diagnostic (niveau de preuve $\mathrm{C}$ ). 


\section{SAHOS}

\section{A. Conduite automobile}

1. Il existe un risque accru de collisions de véhicules à moteurs chez les patients souffrant de SAHOS non traités (niveau de preuve B).

2. Il est difficile de prédire le risque de collisions de véhicules à moteurs chez les patients souffrant de SAHOS en raison des nombreux facteurs confondants (sommeil préalable, travail posté, médicaments et respect du traitement médical) qui peuvent influencer le niveau de risque (niveau de preuve C).

3. Bien que tous les automobilistes aient une obligation individuelle et réglementaire de conduire prudemment et d'éviter tout préjudice prévisible à autrui, les médecins doivent aviser tous les patients souffrant de SAHOS des dangers de la conduite en état de somnolence (niveau de preuve D).

4. Les médecins doivent signaler les automobilistes somnolents aux autorités responsables des véhicules à moteur, dans le respect de la législation locale (niveau de preuve D).

5. Les patients souffrant de SAHOS peuvent reprendre le volant sans danger une fois un traitement approprié mis en place, dans le respect des règlements locaux (niveau de preuve C).

B. Recours aux soins de santé et impact économique

1. Les patients souffrant de SAHOS génèrent des dépenses en soins de santé accrues pendant de nombreuses années avant d'être diagnostiqués (niveau de preuve C).

2. Le SHS est le type de TRS associé aux dépenses en soins de santé les plus élevées, car ces patients doivent souvent être hospitalisés (niveau de preuve C).

3. La ventilation spontanée par pression positive continue (PPC) communément appelé continuous airway pressure (CPAP) réduit les dépenses en soins de santé pendant les deux premières années après un diagnostic de SAHOS (niveau de preuve C).

4. Le traitement du SAHOS constitue une utilisation rentable des ressources de soins de santé (niveau de preuve $\mathrm{B})$.

\section{Traitement comportemental et pharmacologique}

1. Tous les patients obèses souffrant de SAHOS doivent se voir encourager à perdre du poids, mais les tentatives de perte de poids ne doivent pas retarder l'initiation d'un traitement supplémentaire, le cas échéant (niveau de preuve B).

2. Les patients doivent être avisés du fait que l'alcool et les sédatifs peuvent exacerber le SAHOS (niveau de preuve C).

3. Le soulagement de l'obstruction nasale ne doit pas être considéré comme un traitement primaire du SAHOS, mais comme une mesure d'appoint pour faciliter le traitement efficace par PPC ou appareil buccal (niveau de preuve C).
4. Les patients souffrant de SAHOS associé au décubitus dorsal peuvent retirer un important avantage clinique de la thérapie positionnelle (niveau de preuve C).

5. Les agents pharmacologiques (p. ex., inhibiteurs de recaptation de la sérotonine et progestérone) ne constituent pas des thérapies efficaces pour le SAHOS (niveau de preuve B).

6. Les agents promoteurs de la vigilance peuvent constituer un appoint utile pour traiter les patients qui demeurent somnolents malgré une hygiène de sommeil adéquate et qui utilisent un traitement efficace du SAHOS (niveau de preuve B).

D. Quels patients doivent être traités et comment doiventils être suivis?

1. Tous les patients souffrant de SAHOS doivent se voir proposer un essai de traitement pour améliorer leurs symptômes (niveau de preuve B).

2. Les indications de traitement pour les patients asymptomatiques ayant des résultats d'étude de sommeil anormaux ne sont pas claires. Un traitement peut être envisagé pour les patients asymptomatiques ayant des facteurs de comorbidité, qui ont un travail à haut risque personnel ou pour autrui ou dont l'indice d'apnée/hypopnée est supérieur à 30 événements par heure (niveau de preuve C).

3. L'observance au traitement doit être évaluée dans un délai de deux à quatre semaines de l'initiation de celui-ci (niveau de preuve D).

4. Les patients qui ont entamé un traitement doivent être suivis dans un délai de trois mois par un médecin ou un autre prestataire supervisé par un médecin afin d'évaluer l'évolution de leurs symptômes et l'observance au traitement (niveau de preuve D).

5. Un suivi à long terme par un prestataire de soins primaires ou un spécialiste des troubles du sommeil doit être organisé, tout comme pour les autres maladies chroniques (niveau de preuve D).

6. L'éducation des patients quant à la nature, aux complications et au traitement du SAHOS, qui doit être effectuée par un professionnel de la santé qualifié (thérapeute respiratoire / infirmier / technicien en polysomnographie), constitue un élément important de toutes les stratégies de traitement (niveau de preuve $\mathrm{D}$ ).

E. Thérapie par pression positive continue (PPC) (communément appelée continuous airway pressure [CPAP]).

1. La thérapie par PPC classique à pression fixe constitue le principal traitement des patients souffrant de SAHOS (niveau de preuve B).

2. La thérapie par PPC automatique constitue un traitement efficace du SAHOS en l'absence de comorbidités, mais l'efficacité du traitement peut varier d'un appareil à l'autre (niveau de preuve B).

3. Il n'est pas certain que les patients profitent du coût supplémentaire que représente la thérapie par PPC 
automatique par rapport à la thérapie par PPC à pression fixe (niveau de preuve B).

4. Une titration de la PPC au cours d'un enregistrement polysomnographique demeure la norme acceptée pour déterminer la pression optimale de celle-ci (niveau de preuve D).

5. La pression optimale de la thérapie par PPC peut également être déterminée sur la base du profil de pression provenant d'un essai de traitement par PPC automatique, si les conditions d'enregistrement sont évaluées soigneusement et si le profil de pression est analysé en détail (niveau de preuve B).

6. En cas de faible utilisation du traitement par PPC (moins de quatre heures par nuit) pendant deux mois, après des efforts pour améliorer le confort du patient, le traitement doit être reconsidéré (niveau de preuve D).

7. Le traitement par PPC ne doit pas être abandonnée sans avoir tenu compte des facteurs suivants (niveau de preuve D) :

a. avis d'un professionnel de la santé qualifié dans ce type de traitement (c.-à-d., inhalothérapeute / infirmièr[e]);

b. étude de titration au cours d'un enregistrement polysomnographique ou éssai de traitement par PPC automatique pour détecter d'éventuels problèmes;

c. recours à un humidificateur chauffant.

8. La ventilation à deux niveaux de pression (communément appelée bipap ou bi-level) ne doit pas être utilisée de façon courante dans le traitement du SAHOS, mais réservée aux patients souffrant de SHS ou qui ne tolèrent pas le traitement par PPC classique ou automatique (niveau de preuve $\mathrm{D}$ ).

\section{F. Appareils buccaux}

1. Les appareils buccaux constituent une thérapie de première ligne appropriée pour les patients souffrant de SAHOS léger à modéré et ne présentant que des symptômes diurnes minimes (niveau de preuve A).

2. Les appareils buccaux constituent une thérapie alternative appropriée pour les patients qui ne tolèrent pas la thérapie par PPC (niveau de preuve $A$ ).

3. Les appareils buccaux doivent être ajustés par des praticiens dentaires spécialement formés dans les TRS (niveau de preuve D).

4. L'utilisation d'appareils buccaux doit être contrôlée cliniquement après l'initiation du traitement pour permettre l'ajustement de l'appareil et l'évaluation des symptômes et des effets secondaires (niveau de preuve D).

5. Les patients doivent subir une étude du sommeil de suivi avec l'appareil buccal pour vérifier l'efficacité du traitement (niveau de preuve D).

6. Les patients qui ont entamé un traitement par appareil buccal doivent être suivis régulièrement par un praticien dentaire qualifié pendant la première année, puis tous les ans par la suite, pour contrôler l'observance au traitement, la détérioration de l'appareil et la santé bucco-dentaire (niveau de preuve D).

\section{G. Chirurgie des voies aériennes supérieures}

1. La présence d'une hypertrophie amygdalienne chez un patient souffrant de SAHOS justifie une consultation avec un oto-rhino-laryngologiste pour une éventuelle amygdalectomie (niveau de preuve D).

2. Le SAHOS doit être exclu chez les patients qui se voient proposer une opération des voies aériennes supérieures pour cause de ronflement (niveau de preuve C).

3. Les patients qui se voient proposer une opération du palais pour le ronflement doivent être informés des taux d'échec et de réussite de la procédure envisagée et du risque de problèmes d'utilisation du traitement par PPC s'ils développent un SAHOS par la suite (niveau de preuve D).

4. L'uvulopalatoplastie au laser n'est pas recommandée pour le traitement du SAHOS (niveau de preuve B).

5. L'uvulopalatopharyngoplastie peut être envisagée chez certains patients souffrant de SAHOS qui n'ont pas été soulagés par le traitement PPC et/ou par un appareil buccal (niveau de preuve D).

6. La chirurgie maxillomandibulaire peut être efficace pour certains patients souffrant de SAHOS soigneusement sélectionnés qui n'ont pas été traités efficacement par la PPC et/ou par un appareil buccal (niveau de preuve C).

7. La trachéostomie ne doit être envisagée que pour certains patients souffrant de SAHOS soigneusement sélectionnés après l'échec de tous les autres traitements (niveau de preuve D).

8. Pour la plupart des formes de chirurgie des voies aériennes supérieures, les bénéfices dans le traitement du SAHOS n'ont pas été démontrés par des essais cliniques contrôlés. Les procédures nouvelles ou non validées doivent être considérées comme expérimentales et être rigoureusement testées par des études de recherches avant d'être utilisées à grande échelle en pratique clinique (niveau de preuve C).

\section{H. Anesthésie}

1. Les patients souffrant de SAHOS courent un risque accru d'intubation endotrachéale difficile (niveau de preuve $\mathrm{C}$ ).

2. Les médicaments administrés pendant l'anesthésie et la période postopératoire peuvent augmenter la gravité du SAHOS après une opération (niveau de preuve C).

3. Les patients souffrant de SAHOS doivent débuter un traitement avant toute opération. Lorsqu'un patient est traité par PPC avant une opération, cette thérapie doit être continuée immédiatement après l'opération (niveau de preuve D). 
4. Tous les patients présentant un risque de complications respiratoires dues au SAHOS doivent être surveillés par oxymétrie après leur opération. Les patients qui présentent un risque accru d'ischémie ou d'arythmie cardiaque doivent également subir une surveillance cardiaque (niveau de preuve D).

5. Le traitement par PPC ne doit pas remplacer une surveillance postopératoire adéquate (niveau de preuve D).

\section{SAHSC/RCS}

1. La RCS est associée à une mortalité accrue chez les patients souffrant d'insuffisance cardiaque (niveau de preuve B).

2. Un traitement médical optimal constitue la première ligne du traitement de la RCS chez les patients souffrant d'insuffisance cardiaque (niveau de preuve C).

3. La thérapie par PPC et/ou l'oxygène ne sont pas recommandés comme thérapie de routine pour les patients souffrant de RCS et d'insuffisance cardiaque (niveau de preuve B).

4. Il n'existe aucun traitement prouvé efficace pour le SAHSC idiopathique (niveau de preuve D).

\section{SHS}

1. Tous les patients souffrant de cas présumés de SHS doivent être adressés en urgence à un spécialiste pour évaluation et suivi à long terme (niveau de preuve D).

2. La thérapie par PPC constitue le traitement principal des patients souffrant de SHS en cas d'obstruction associée des voies aériennes supérieures (niveau de preuve D).

3. La ventilation assistée (pression positive à deux niveaux (communément appelée bi-pap ou bi-level avec ou sans fréquence respiratoire minimale imposée, et respirateurs à pression et volumétriques) doit être envisagée si le traitement par PPC n'améliore pas les échanges gazeux diurnes et nocturnes (niveau de preuve D).

4. L'oxygène doit être envisagé chez les patients souffrant d'hypoxémie persistante malgré un traitement par PPC ou par ventilation assistée (niveau de preuve D).

5. Le traitement doit être initié en unité de soins intensifs, en laboratoire de sommeil ou dans un autre environnement étroitement surveillé offrant un soutien approprié pour garantir la mise en œuvre sécuritaire et efficace de la thérapie par PPC, de la ventilation assistée et de l'oxygène (niveau de preuve D).

6. L'efficacité du traitement doit être vérifiée par une polysomnographie sous surveillance et une analyse des gaz sanguins artériels afin d'évaluer objectivement l'impact sur les échanges gazeux diurnes et nocturnes (niveau de preuve D).

\section{LECTURES RECOMMANDÉES}

1. American Academy of Sleep Medicine. International Classification of Sleep Disorders, 2nd edn: Diagnostic and Coding Manual.

Westchester: American Academy of Sleep Medicine, 2005.

2. American Society of Anesthesiologists. Practice Guidelines for the Perioperative Management of Patients with Obstructive Sleep Apnea. $<$ http://www.asahq.org/publicationsAndServices/practiceparam.htm\#a pnea> (Version current at July 5, 2006).
3. American Society of Anesthesiologists Task Force on Management of the Difficult Airway. Practice guidelines for management of the difficult airway: An updated report by the American Society of Anesthesiologists Task Force on Management of the Difficult Airway. Anesthesiology 2003;98:1269-77. (Erratum in 2004;101:565).

4. ATS/ACCP/AASM Taskforce Steering Committee. Executive summary on the systematic review and practice parameters for portable monitoring in the investigation of suspected sleep apnea in adults. Am J Respir Crit Care Med 2004;169:1160-3.

5. Centers for Medicare \& Medicaid Services. Medicare coverage database. <https://www.cms.hhs.gov/mcd/viewdecisionmemo.asp> (Version current at July 5, 2006).

6. Chesson AL Jr, Berry RB, Pack A; American Academy of Sleep Medicine; American Thoracic Society; American College of Chest Physicians. Practice parameters for the use of portable monitoring devices in the investigation of suspected obstructive sleep apnea in adults. Sleep 2003;26:907-13.

7. College of Physicians and Surgeons of Ontario. Clinical Practice Parameters and Facility Standards for Sleep Medicine, 2nd edn. Toronto: College of Physicians and Surgeons of Ontario, 2005.

8. Ferguson KA, Cartwright R, Rogers R, Schmidt-Nowara W. Oral appliances for snoring and obstructive sleep apnea: A review. Sleep 2006;29:244-62.

9. Flemons WW, Littner MR, Rowley JA, et al. Home diagnosis of sleep apnea: A systematic review of the literature. An evidence review cosponsored by the American Academy of Sleep Medicine, the American College of Chest Physicians, and the American Thoracic Society. Chest 2003;124:1543-79.

10. George CF. Standards for polysomnography in Canada. The Standards Committees of the Canadian Sleep Society and the Canadian Thoracic Society. CMAJ 1996;155:1673-8.

11. Giles TL, Lasserson TJ, Smith BJ, White J, Wright J, Cates CJ. Continuous positive airways pressure for obstructive sleep apnoea in adults. In: The Cochrane Library, Issue 1, 2006. Chichester, UK: John Wiley \& Sons, Ltd.

12. Haniffa M, Lasserson TJ, Smith I. Interventions to improve compliance with continuous positive airway pressure for obstructive sleep apnoea. In: The Cochrane Library, Issue 2, 2006. Chichester, UK: John Wiley \& Sons, Ltd.

13. Kushida CA, Littner MR, Morgenthaler T, et al. Practice parameters for the indications for polysomnography and related procedures: An update for 2005. Sleep 2005;28:499-521.

14. Kushida CA, Morgenthaler TI, Littner MR, et al; American Academy of Sleep. Practice parameters for the treatment of snoring and obstructive sleep apnea with oral appliances: An update for 2005. Sleep 2006;29:240-3.

15. Lim J, Lasserson TJ, Fleetham J, Wright J. Oral appliances for obstructive sleep apnoea. In: The Cochrane Library, Issue 1, 2006. Chichester, UK: John Wiley \& Sons, Ltd.

16. Loube DI, Gay PC, Strohl KP, Pack AI, White DP, Collop NA. Indications for positive airway pressure treatment of adult obstructive sleep apnea patients: A consensus statement. Chest 1999;115:863-6.

17. Meoli AL, Casey KR, Clark RW, et al; Clinical Practice Review Committee. Hypopnea in sleep-disordered breathing in adults. Sleep 2001;24:469-70

18. Meoli AL, Rosen CL, Kristo D, et al; Clinical Practice Review Committee; American Academy of Sleep Medicine. Upper airway management of the adult patient with obstructive sleep apnea in the perioperative period - Avoiding complications. Sleep 2003;26:1060-5.

19. Practice parameters for the indications for polysomnography and related procedures. Polysomnography Task Force, American Sleep Disorders Association Standards of Practice Committee. Sleep 1997;20:406-22.

20. Scottish Intercollegiate/British Thoracic Society Guideline. Management of obstructive sleep apnoea/hypopnoea syndrome in adults. Guideline No 73, 2003.

<http://www.sign.ac.uk/guidelines/fulltext/73/index.html> (Version current at July 5, 2006).

21. Shneerson J, Wright J. Lifestyle modification for obstructive sleep apnoea. In: The Cochrane Library, Issue 2, 2006. Chichester, UK: John Wiley \& Sons, Ltd.

22. Sleep-related breathing disorders in adults: Recommendations for syndrome definition and measurement techniques in clinical research. The Report of an American Academy of Sleep Medicine Task Force. Sleep 1999;22:667-89.

23. Smith I, Lasserson TJ, Wright J. Drug treatments for obstructive sleep apnoea. In: The Cochrane Library, Issue 2, 2006. Chichester, UK: John Wiley \& Sons, Ltd.

24. Sundaram S, Bridgman SA, Lim J, Lasserson TJ. Surgery for obstructive sleep apnoea. In: The Cochrane Library, Issue 2, 2006. Chichester, UK: John Wiley \& Sons, Ltd. 


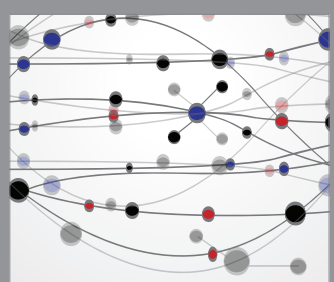

The Scientific World Journal
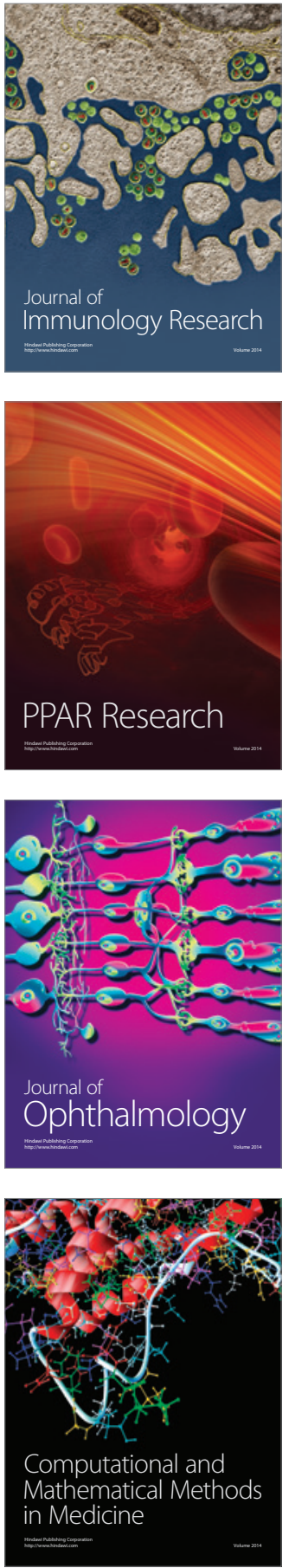

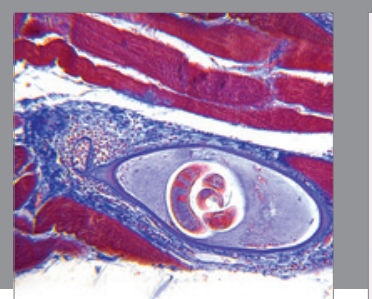

Gastroenterology Research and Practice

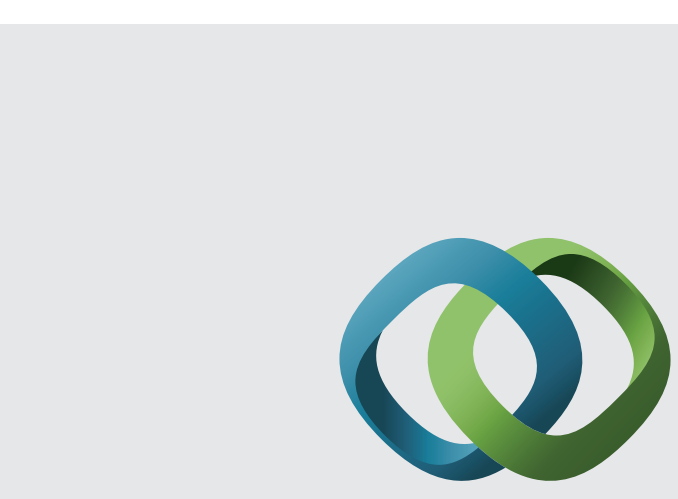

\section{Hindawi}

Submit your manuscripts at

http://www.hindawi.com
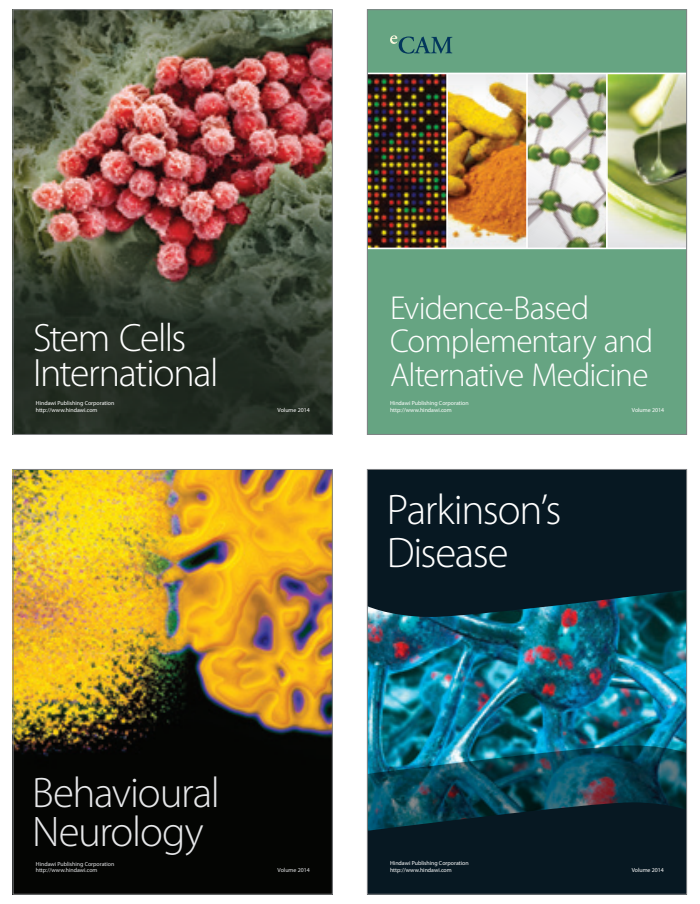
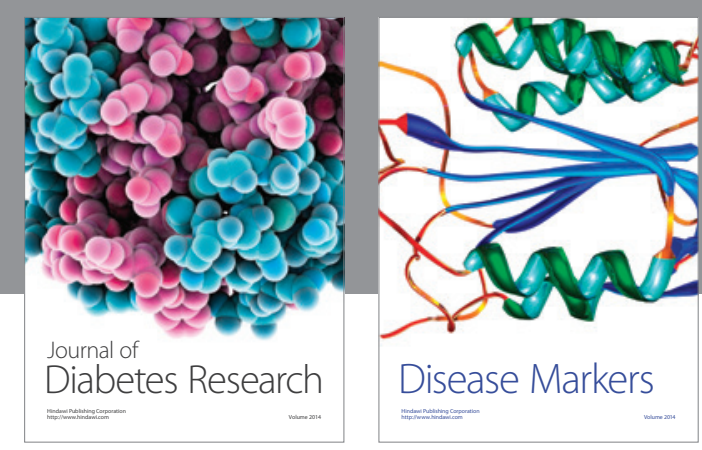

Disease Markers
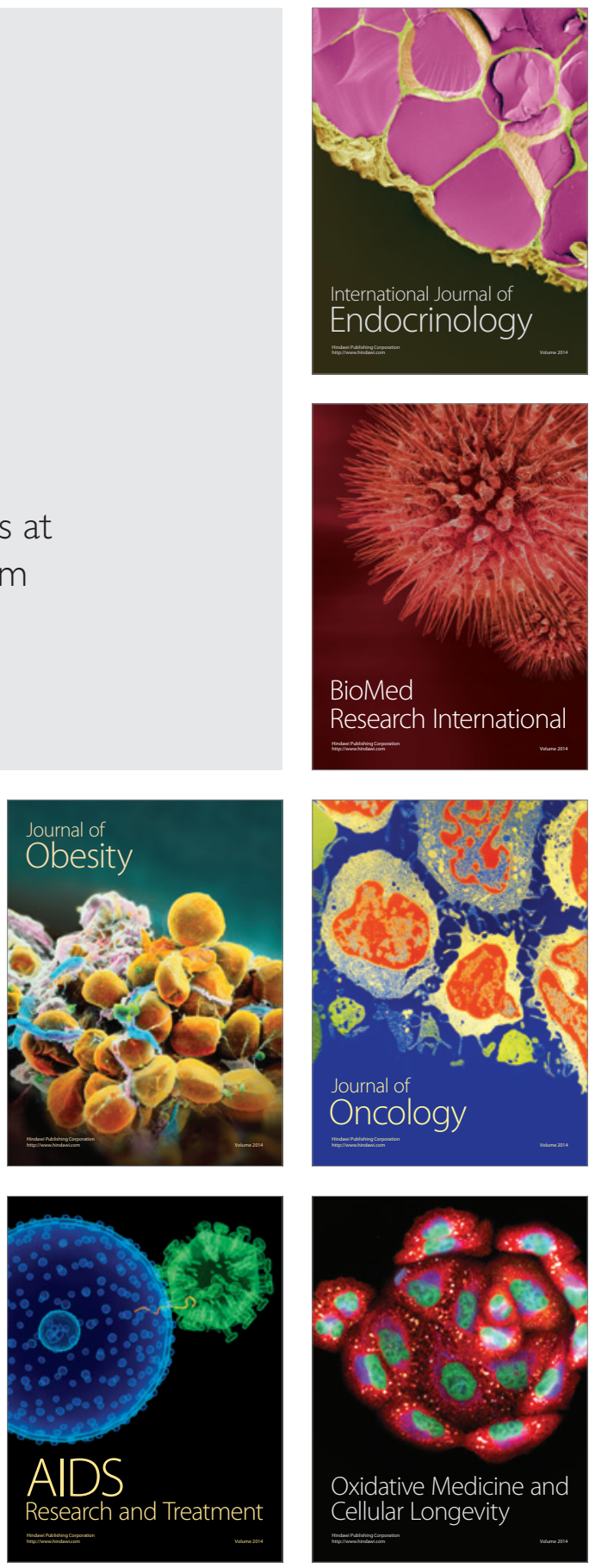